\title{
A randomized EPIREMED protocol study on the long-term visuo spatial effects of very preterm children with a working memory deficit
}

Catherine Gire ${ }^{1,2}$, Any Beltran Anzola ${ }^{1,2^{*}}$ (D), Monique Kaminski ${ }^{3}$, Karine Baumstarck ${ }^{2}$, Pierre-Yves Ancel ${ }^{4,5}$, Julie Berbis ${ }^{2}$ and for EPIREMED-Study Group

\begin{abstract}
Background: Very preterm children generally perform poorly in executive functions and particularly in working memory. Adaptive training tasks encouraging these children to work continuously on their personal working memory capacity can be very useful. Above all in preschool-age children, several cognitive training programs focused on improving working memory capacity. Cogmed is a computerized visuospatial cognitive training program that improves working memory in children and adolescents with attention-deficit/hyperactivity disorder. The main objective is to assess the long-term effects (18 months) of cognitive training (Cogmed) on visuospatial processing in preschool-age very preterm children with working memory impairment.

Methods: The EPIREMED study is a prospective, randomized, controlled, multicentric trial nested in a population based epidemiological survey. An intervention group (Cogmed cognitive training) and a control group (standard care management) will compare children aged 51/2 to 6 years, born between 24- and 34-weeks' gestational age, with a global intelligence quotient $>70$ and a working memory index $<85$. The study will include 166 children from national study EPIPAGE-2 (Epidemiological Study on Small Gestational Ages). The intervention consists of 25 sessions administered over a 5- to 8-week period. The primary endpoint will be the visuospatial processing, assessed by the score of the visuospatial index: score of the WPPSI-IV (Wechsler Preschool and Primary Scale of Intelligence). The secondary endpoints will allow to assess the executive functions, language and abilities, infant behavior, quality of life assessment, school performance and parental anxiety.
\end{abstract}

\footnotetext{
* Correspondence: any-alejandra.beltran-anzola@univ-amu.fr

${ }^{1}$ Department of Neonatology, North Hospital, APHM University Hospital, Marseille, France

${ }^{2}$ CEReSS - Health Service Research and Quality of Life Center, Faculty of

Medicine, Aix-Marseille University, 27 Bd Jean Moulin, 13385 Marseille, cedex 05, France

Full list of author information is available at the end of the article
}

(c) The Author(s). 2021 Open Access This article is licensed under a Creative Commons Attribution 4.0 International License, which permits use, sharing, adaptation, distribution and reproduction in any medium or format, as long as you give appropriate credit to the original author(s) and the source, provide a link to the Creative Commons licence, and indicate if changes were made. The images or other third party material in this article are included in the article's Creative Commons licence, unless indicated otherwise in a credit line to the material. If material is not included in the article's Creative Commons licence and your intended use is not permitted by statutory regulation or exceeds the permitted use, you will need to obtain permission directly from the copyright holder. To view a copy of this licence, visit http://creativecommons.org/licenses/by/4.0/ The Creative Commons Public Domain Dedication waiver (http://creativecommons.org/publicdomain/zero/1.0/) applies to the data made available in this article, unless otherwise stated in a credit line to the data. 
Discussion: This project's primary goal is to demonstrate the necessity of early visuospatial memory assessment within the vulnerable population of very preterm children, and to prove the feasibility and efficacy of computerized cognitive training using online software programs. A better global neuropsychological development improvement (visuospatial processing and other far transfer) can be expected with an improvement in learning and decreased behavioral problems. In the long term, these improvements might also reduce those global costs linked to the consequences of extreme prematurity.

Trial registration: NCT02757794 (registered on 2nd May 2016 at ClinicalTrial.gov).

Keywords: Very preterm children, Working memory, Visuospatial index, Cognitive training, Executive functions

\section{Background}

Very Preterm (VP) birth rates and survival rates have risen in France $[1,2]$. However, the proportion of VP survivors with severe deficits has remained stable. Neuropsychological disorders and/or behavioral deficits are the most frequently encountered deficiencies [2-8] and have serious consequences on learning [9], familial and social adaptations as well as an impact on the child's future adult years [10].

\section{Executive function}

Executive Functions define the cognitive operations that allow an individual to adjust behavior and activity in response to environmental requirements and fluctuations.

Compared to peers born at term, VP children generally perform poorly in Executive Functions (EF) and, in particular, in Working Memory (WM).

The EF comes into play when an individual is confronted with a "non-routine" situation which requires problem solving. The principal mental processes characterizing EF are:

- organizing and planning data based on what needs to be achieved, choosing relevant information,

- implementing processing operations, inventing new situations and modifying them if they deviate from the (original) purpose [11, 12],

- suppressing extraneous information, resisting distractions,

- organizing task-relevant information in their memory for later use (WM).

While each of these mental processes or "executive mental functions" can be evaluated by "specific" tests, they are often interlinked and dependent on the mental attention processes (auditory and/or visual). For this reason, definitions vary significantly from publication to publication $[11,13]$.

Schematically we can isolate four main executive mental processes [14]:
1. Planning: mental diagram of a single action, anticipating the goal to be reached - "how to achieve that goal".

2. Flexibility: adaptation of an action plan for environmental contingencies, ability to modify strategies in case of error, maintain attention - "the art of adapting to change".

3. Inhibition: capacity to ignore distractions and to resist giving one reply rather than another.

4. Working Memory: ability to store verbal or visuospatial information in one's mental space and to manipulate it, implementing strategies, processing action sequences, reasoning - "art de faire". (Having the knack)

\section{Executive function and very preterm}

There is a considerable interest in EF in very preterm children $[15,16]$. In studies published between 1990 and 2008 a systematic review performed by Mulder et al., analyzed the executive process in 830 very prematurely born school children vs 740 children born full-term [15]. The authors showed a difference of 0.3 Standard Deviation (SD) for inhibition, $0.5 \mathrm{SD}$ for verbal Working Memory (WM) and 0.4 SD for planning skills as compared to the full-term control group. These differences were even greater for those with lesser gestational ages: $0.5 \mathrm{SD}$ for the inhibition and 0.7 SD for verbal WM within 26 weeks GA (weeks' amenorrhea).

This gap is interesting, especially for verbal WM, and it increases with the age of children suggesting a worsening over time. Mental flexibility remains unchanged as compared to the control group. The Aarnoudse-Moens's meta-analysis involved children born between 1998 and 2008 comparing very extremely premature births to fullterm births [16]. This meta-analysis showed a decrease of 0.6 SD for verbal WM and 0.4 SD for the visuospatial WM. Unlike Mulder et al., this difference is shown to stabilize with age, and their mental flexibility is impacted $0.5 \mathrm{SD}$ as compared with the control group.

In 2018-2019, Brydges' meta-analysis of 60 studies included a total of 6163 preterm children born before 32 
weeks' amenorrhea and a control group of 5471 fullterm children. The children in both groups were between 4 and 17 years of age [17]. Altogether, VP children obtained a result of -0.51 SD (95\% CI $0.44-0.58 ; p<$ 0.001 ) for executive functions. Van Houdt, in 2019, compiled 35 studies (3360 preterm and 2812 full-term infants) and reported an overall deficit in EFs in VP children of approximately - 0.5 SD [18]. The study observed a deficit of $-0.52 \mathrm{SD}$ in working memory, -0.39 SD in inhibitory control, and -0.51 SD in flexibility. However, the deficit difference in each domain was not significant. All three EFs are affected to the same extent in VP children.

These data are corroborated in the meta-analyses of Twilhaar in 2018 [19] regarding academic performance (17 studies, 2390 children, - 0.71 SD in mathematics, $0.44 \mathrm{SD}$ in reading and $-0.52 \mathrm{SD}$ in spelling). Allotey in 2018 reported -0.67 SD in reading, -0.56 SD in spelling and $-0.78 \mathrm{SD}$ in mathematics amongst VP children aged 5-to-8- years [6]. Additionally, Allotey pointed out that this difference in academic results continued to persist in secondary education. The latest meta-analysis of 33 studies, which covered 4000 premature infants, confirms this academic gap in math and reading performances between VP children and their peers [20]. Two metaanalyses looked at the evolving profile of executive deficit in extremely premature infants $[18,21]$. The last, by van Houdt, suggested a stability of this deficit, at least over studied ages of $4 \frac{1}{2}$ and 14 years of age. The authors do not exclude possible modifications afterwards, at the end of adolescence or in adulthood, in view of the slow maturation of EFs.

\section{Working memory}

WM is defined as a "brain system that provides temporary storage and manipulation of information necessary for complex cognitive tasks" [22]. WM is regulated by a central executive control system and two subordinate subsystems: the visuospatial sketchpad and the phonological loop. This process is considered as a prerequisite for other EFs such as reasoning and planning and for predicting intelligence and academic success. In VP children, WM impairment is linked to learning disabilities and is reported to have a strong influence on language and visuospatial processing [23]. Very premature-birth children present with frequent WM deficits [16, 24, 25]. Indeed, the literature shows that visuospatial WM is not as good in VP preschool children as term birth children [26-28]. In 2014, Omizollo et al., studied the correlation between WM (verbal and visuospatial) and the learning in a cohort group of seven-year-olds very prematurely born. The VP group had 2.1 to 3.5 times more deficit in WM than the control group [29]. In these school age children, WM might be correlated with subsequent learning disorders and the origin of complex deficits such as language delays or visuospatial performance disorders [15]. Overall academic achievements are thus impaired and an intervention strategy to minimize prematurity's long-term WM impact needs to be developed.

\section{Cognitive training}

In recent years, several Cognitive Training (CT) programs have focused on improving WM capacity by adaptive training tasks that encourage individuals to work continuously on their personal working memory capacity. Many re-education techniques (books, games, software, etc.) have emerged in recent years without any "gold standard". Cogmed JM [30-32], for preschoolers ages four to six, is a technique for reeducating the visual spatial WM and is used in many randomized studies of CTs, WMs and EFs [33]. This computer software has a specific set of visuospatial memory tasks and adjustable levels of difficulty by using a precise algorithm. These programs have succeeded in improving individual performance in some specific WM capacities, but not in other everyday EF functions such as, language and visuospatial processing $[11,34,35]$. Therefore, the functional benefits of CT have become controversial. Recently one metanalysis showed that cognitive training programs for preschoolers are significantly more effective for developmentally at-risk children (ADHD or low socioeconomic status) than for children with typical development and without risks. This metanalysis assessed also other factors as the individual vs collective session and the training duration; these two factors were considered significant moderators contrary to the number of sessions and the computerized against non-computerized training [36]. The efficacy of Cogmed was particularly studied by two scientific publications, Shinaver et al. and Spencer-Smith et al. [33, 37]. The impact on the trained WM visual spatial is significant and seems to be sustained over time. Improving non-trained functions such as verbal WM, attention, and secondary learning disorders, are possible, but have yet to be proven on larger numbers. Above all the WM cognitive training studies of preschool age children were realized with Cogmed JM [30-32].

\section{Cognitive training and prematurity}

Three studies on premature infants have examined the effects of CT using Cogmed on visuospatial WM [3840]. The first was a sample of 16 preterm vs. 19 termbirth teenagers. The second was performed on $20 \mathrm{VP}$ preschool children ages 5-6 years and the third study included $20 \mathrm{VP}$ preschool children vs 17 term births. 
There appears to be a beneficial effect on the trained visuospatial WM and a possible transfer to other processes (verbal working memory, attention, etc.). However, these three studies contained very small samples from very different age groups. In two studies the WM was in the process of maturation, and in the third it was practically consolidated. Although, monitoring was limited to 7 months and learning disorders were not assessed, they nonetheless represented preliminary studies with encouraging results.

The IMPRINT study [41, 42] is the only large, doubleblind, randomized study using a Cogmed intervention group and a control group (Cogmed placebo without TM training). The study includes 126 seven-year-old, VP children $(<28$ SA, $<1000 \mathrm{~g}$, with no cognitive deficits, and having a median IQ 95). The IMPRINT study shows that only the working memory at 2 weeks post intervention was improved in the intervention arm. This did not persist at the first and second year after training. There was no benefit from the Cogmed training on their executive behaviour, their attention or their school results, either at 2 weeks after the intervention or after 1 to 2 years after the intervention.

The authors concluded that Cogmed was not recommended for improving academic performances for VPs at this age (7 years) [42]. Thus, it seems that for VP children, the Cogmed computerized WM training program can provide an immediate and direct WM benefit but no immediate transfer and no long-term benefits.

As executive disorders are the core of VP neurodevelopmental problems (learning difficulties, attention disorders, behavioral disorders) and are identified in VP children, it raises the question of possible cognitive training of executive functions to reduce problems and improve the daily quality of life for the children and their parents. Once the concept of executive training is put forward, the questions are: the temporality of the training and at what age will this executive training be most relevant? What are the precise objectives of this training and how will it be evaluated? Finally, the precise modalities (duration, type of exercise, chosen medium, etc.) of the training program need to be defined to maximize its effectiveness [43].

\section{Hypothesis}

Even if some literature data exist on Cogmed's CT visuospatial WM effects in premature infants there are some limits that need to be discussed. The sample size and compliance of training from participants might influence the robustness of the results. In fact, those elements of these studies are generally modest (below or about 50 patients randomized in interventional group and around 20 patients with good training compliance) [38-42]. Furthermore, none of these studies have been carried out in France where the aspects of the French healthcare system are very specific. Moreover, the efficacy of visuospatial CT might be modified according to the age at which children perform the CT. In fact, visuospatial CT is a well-adapted method to reach WM in children 5-to-6-years old whose WM is only a visuospatial (subcomponent visuospatial sketchpad), taking advantage of the neuroplasticity period. The central executive control system and the phonological loop will develop in children later on. Furthermore about 30\% of VP children have low performances on their visuospatial processing. Thus, a visuospatial CT for 5-to-6-year olds will enable a measurement of the consequences of visual spatial WM restoration on the overall brain function and learning in VP children [38, 39].

We hypothesize that CT with preschool VP children (just as their WM is emerging and uniquely non-verbal: visuospatial sketchpad) may decrease the subsequent dysexecutive disorders, improve intellectual performance (both visuospatial and language processing) as well as school integration. This study will therefore assess CT ability to improve visuospatial processing and moreover to assess its impact on the global function and learning abilities of the brain.

\section{Objectives}

Our primary objective is to assess the long-term effects (18-months post inclusion [+/-2 months]) of Cognitive Training (Cogmed JM) on the visual-spatial processing in VP preschool-aged children with WM impairment. Visuospatial processing is a broad cognitive process encompassing many subcomponents such as attention, sensory-motor skills, EF and visuospatial WM.

The secondary objectives are to assess the effects of the cognitive training on the following parameters at the six-month post-intervention (+/- 2 months):

Children' parameters:

- Global intellectual functioning,

- different cognitive processes: working memory,

- language, visual-spatial processing, speed processing,, and fluidity of intelligence,

- other composites of executive functions: auditory attention, flexibility, and inhibition,

- language processing abilities: verbal learning abilities (cultural and cognitive), phonological judgment and semantics, verbal processing speed, verbal WM, motor programming, visual attention, and ability to analogize,

- behavior and quality of life (QoL),

- school performance.

Parents' parameters: 
- Anxiety level.

\section{Methods/design Study design}

This study is designed as a multicenter (18 units of French university hospitals), randomized, controlled, open-label, two-parallel groups study. The recruitment will be prospective. The two groups are:

- A control group: standard care management,

- an experimental group: standard care management in association with a 2-month Cognitive Training program called Cogmed JM.

The list of the recruiting centers is availed in website clinicaltrial.gov

\section{Participants}

Participants must meet all of the following criteria:

\section{Inclusion criteria}

- children already included in the EPIPAGE 2, born between 24-and 34-weeks' gestational amenorrhea,

- children aged $5 \frac{1}{2}$ to 6 years old,

- children exhibiting a total intellectual quotient $>70$ from the Wechsler Pre-Primary Scale of Intelligence - Fourth Edition (WPPSI-IV) (during the 5-year assessment in EPIPAGE 2),

- children having a visuospatial WM impairment defined by a working memory index $<=85$ from the WPPSI-IV,

- children with parents (or legal guardians) authorizing participation in the study and a signed informed consent form,

- children with medical insurance.

\section{Non-inclusion criteria}

- Children with severe cerebral palsy, based on the Gross Motor Function (score $>2$ ) and Bimanual Fine Motor Function (score >) 2 classification system [44, 45],

- children with blindness or amblyopia, defined by a visual acuity $<3$ (during the 5 -year assessment in EPIPAGE 2),

- children with deafness, as defined by a prescribed hearing aid,

- children with chromosomal disorder or autistic syndrome,

- children included in the EPILANG study protocol (VP children with language delay and parent intervention [Effectiveness of speech-language pathology parental guidance for very preterm infants with language delay] (an ancillary project to EPIPAGE 2),

- children who do not speak French,

- children with parents having no internet connection,

- triplets.

\section{Exclusion criteria}

- Children and / or parents wishing to interrupt his / her participation during the study.

\section{Groups}

Experimental group: the cognitive training (CT)

A neuropsychologist or speech therapist will oversee the experimental group's Cogmed training, and build a support structure for both the patient and their parents during the initial interview. Children and their parents will become acquainted with the program which will include a software presentation, the setting of expectations, the CT objectives and establishing a reward system along with a document to explain WM and the software. The program includes a total of three 15-min sessions per week for 8 weeks and involves.

Cogmed JM (4-7-year-olds) is a computerized, online WM rehabilitation program. This will be executed at home, at the hospital or in a rehabilitation center with a "tutor" according to the parents' abilities and their access to an internet connected computer. The child, who will be accompanied by either one or both parents or by a "guardian", will be given a series of interactive, automatically and individually adapted exercises. In Cogmed JM, sessions last 15-20 min, with three exercises out of seven for each session, with a graphic interface.

The Cogmed JM practitioner will consult the on-line compliance and exercise results after each session. The program calculates the performance index: the difference between the maximum level and the starting level which is used to assess progress against a standard norm.

The parents will receive a weekly, 30-min interview in order to support and strengthen their child's motivation. The interview will focus on the child's evolving performance and reward progress.

\section{Control group: current standard care management}

The control group will not be offered a rehabilitation program but rather be followed-up along with their routine care management. Speech therapy and/or academic support may be recommended for those experiencing scholastic difficulties. The control group's visits and questionnaires will be the same as for the parents and the teacher in the experimental group. 


\section{Endpoints}

\section{Primary endpoint}

The visual-spatial processing will be assessed by using the visuospatial index (VSI) of the WPPSI-IV at the inclusion visit, the six-month post-inclusion $(+/-2$ months) and at 18-months post inclusion (+/-2 months). This index consists of two subtests: block design and object assembly. The average score is 100 with a standard deviation of 15 .

Rationale for the primary endpoint The visual perceptual integration testing performance for VPs is poorer than those born full term, and these VPs present with a visuo-constructive dyspraxia. This deficit is connected to a poor integration of visual function, perceptual and/or fine motor skills (e.g., reproduction of a complex geometric figure). This disorder is three times more frequent in VP adolescents than in those born at term. Among those born extremely prematurely, 30\% have results below the 15th percentile in visuospatial performance [46]. The impact of WM rehabilitation on visuospatial skills is an interesting line of research, most particularly in premature infants.

\section{Secondary endpoints}

The secondary endpoints are related to the children and to their parents will be assessed at the inclusion visit and at the six-month post-inclusion (+/-2 months).

\section{Children's endpoints}

- The intellectual functioning and other cognitive processes will be obtained by global intellectual quotient (IQ) and IQ indexes using the WPPSI-IV (Wechsler) [47].

The WPPSI-IV, designed for children ages 4-to-7years-old, assesses the overall intellectual functioning (total comprehensive intellectual quotient) and specific cognitive processes. This is done through the main indices:

- verbal comprehension index,

- fluid reasoning index,

- visual spatial index,

- processing speed index, and

- the working memory index.

The evaluation of the Working Memory Index by the WMI in WPPSI-IV is uniquely visuospatial at this age. The average is 100 with a standard deviation of 15 , as with all Wechsler Scales. The global IQ, as well as the main indices, will be assessed when monitoring the EPIPAGE cohort. It is lower for VP infants as compared to full-term infants [5].
- Executive and attention processes: The NEPSY-2 (NEuroPSYchology assessment - Second Edition) assesses the neuropsychological development of preschool and school age children (3-12 years old) and is used to obtain emerging executive functions in 5-6-year-olds [48]. Auditory attention, statue, and design fluency are the only three subtests in the Executive and Attention Function domains which will be administered in this study. These three tests measure selective and divided attention in auditory modality, inhibition and mental flexibility. A review of Van de Weijer-Bergsma, which is confirmed by Mulder in a meta-analysis, shows that the selective, divided and supported attentional domains are likely to be affected in VP pre-schoolers [15, 49].

- Evaluation of language and its skills: Language is a complex mental process requiring an assessment of all its components. This assessment will be made from the CLéA battery calibrated for those children between 2 and 15-years-old [50].

The battery consists of seven tests:

- Known Digital Channels: a reflection of verbal learning ability, both cultural and cognitive.

- Oral Word Identification: capacity to give a word phonological and semantic judgment.

- Rapid Denomination: timed tested; gives an indication treatment speed.

- Word memory: explores the short-term mnemonic span.

- Facial and oral praxis photographs: relevant in motor programming.

- Visual attention: inspired from NEPSY, it questions the visual spatial component.

- Resolution of logical problems: ability to reason analogically (progressive matrices).

Studies suggest that specific language disorders can be associated with specific WM impairments, particularly with the phonological loop [51, 52]. Two metanalysesstudies demonstrate global language gaps/impairment in the very premature vs. the full-term child with deficits in learning ability, phonology, semantics, grammar, speech coherence and verbal reasoning $[53,54]$.

- Behavioral Evaluation: the child's behavior will be assessed with the Goodman Strengths and Difficulties Questionnaire, which includes 25 selfadministered questions answered by the parents [55], and will assess any impact this intervention has on the child's behavior.

- Evaluation of the child's quality of life (QoL): The quality of life of the children will be assessed using the Perceived Quality of Life and Health of Adolescents and Children Questionnaire (VSP-A 
[Vie et Santé Perçue de l'Adolescent et de l'enfant])

[56] as reported by the parents. The 49-item version portrays nine dimensions and index:

- relationships with parents/family,

- body image,

- vitality,

- relationships with friends,

- general well-being,

- leisure,

- school performance,

- relationships with teacher,

- relationships with medical staff.

A higher score, which ranges between 0 and 100, indicating a better QoL. The French norms are available through Ravens-Sieberer U 2007 [57].

- Schooling: This will be evaluated by the GSA questionnaire (Global School Adaptation score), a French tool completed and validated by the teacher, [58] and re-evaluated in a preschool population in 2013 [59]. The questionnaire covers five verbal skills (verbal communication, verbal participation, vocabulary, syntax, pronunciation), five non-verbal abilities (memory, arithmetic, logical reasoning skills, manual dexterity and fine motor skills) and eight questions evaluating class behavior (compliance with rules, attention, autonomy, speed of accomplishing the task, self-esteem, ability to keep the pace and fatigability). The final question asks the teacher about possible future special educational needs of the child.

\section{Parents' endpoints}

- Anxiety: The Spielberger state-trait anxiety inventory (STAI) will be used to assess anxiety. The STAI is a self-reporting questionnaire consisting of 40 items that measure both the state and trait scores. These scores range from 20 (absence of anxiety) to 80 (high anxiety) [60]. This questionnaire will assess if anxiety is impacted as a result of parental intervention (mother).

Rationale for the secondary endpoints It is of value to measure the impact of intervention on the WM and on other non-trained brain processes as well as on parental anxiety, child behavior and parental perceptions of the child's quality of life.

\section{Participant timeline}

The total participation time is 20 months (Fig. 1).

\section{Sample size}

The sample size was calculated to obtain an $80 \%$ power to detect a difference of 7.5 points on the VSI index (estimated standard deviation: 15) at 18 months $(+/-2$ months) between the two groups. This has been considered to be clinically significant considering other similar studies [5]. With the threshold for statistical significance set at a $p$-value of 0.05 , assuming that a potential $15 \%$ of patients will be lost to follow-up between baseline and last assessments, these calculations showed that 166 patients are needed (83 per group [Power Analysis and Sample Size Software Version 2008, Utah, USA]). Assuming that $30-40 \%$ of the EPIPAGE 2 children will present with a WM abnormality, 1600 children will be required for screening.

\section{Recruitment}

The study will include children in EPIPAGE 2 from the regions participating in the present study (EPIREMED). Recruitment will be at the end of the EPIPAGE 2 assessment (5 $1 / 2$ years). The EPIPAGE 2 protocol and the main perinatal results are described in two publications of Ancel P-Y et al. [2, 61].

The EPIPAGE 2 clinical research coordinator will establish a monthly, per center list of potentially eligible patients to EPIREMED. Using this list, each center's referring staff person will organize the first telephone interface with those qualified families having a WM index lower than 85 , who have no exclusion criteria and who have the ability to travel to the testing centers.

Thereafter, the coordinator will contact those families to further coordinate their EPIREMED participation. The coordinator will explain the study's objectives, its development, and its advantages and disadvantages.

Those families accepting to participate will receive a parent information letter describing all topics discussed by telephone, an information text specifically adapted for a child, an informed consent and a verification of their appointment. Travel expenses will be reimbursed.

\section{Randomization}

A randomization list will be established before the implementation of the study with a 1:1 allocation ratio, and will be elaborated by a secure Clinical Research Platform. A computer-generated, randomized list using a permuted block design will be done (stratified on center and gemellarity: singleton/twin). Multiple births represent $30 \%$ of the preterm population within the EPIPAGE 2 group. If one twin has a WM anomaly, this child will be selected for randomization.

\section{Data collection methods The inclusion visit}

After consents have been obtained from the parent or legal guardian, the following assessment will be obtained:

a) The children 


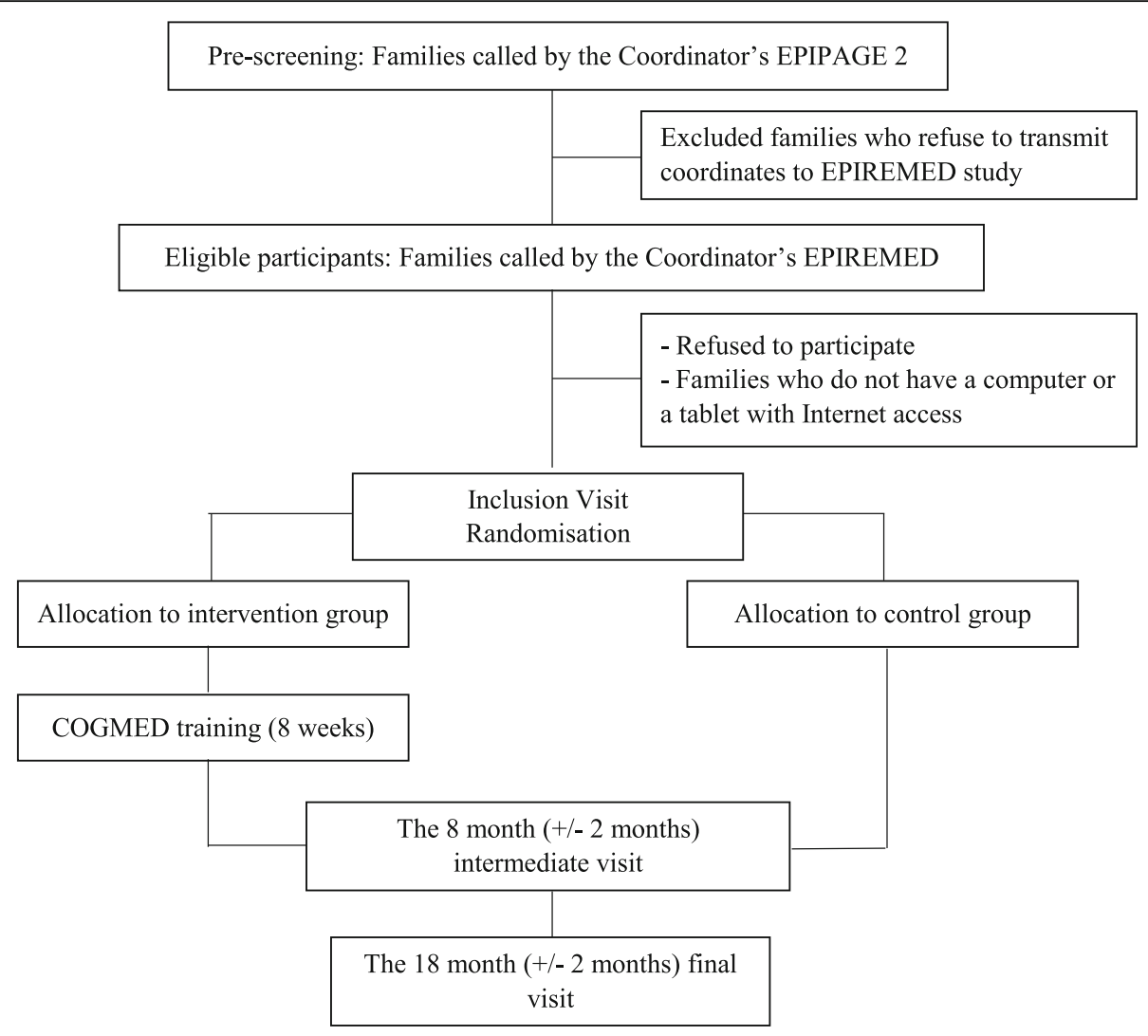

Fig. 1 Flowchart EPIREMED Study

- Assessment of the working memory index (WMI)

- Auditory attention, statue, and design fluency of the NEPSY-2

- Language and verbal skills (CLéA)

This assessment will be made by a neuropsychologist and will last about 90 minutes.

b) The parents:

- A self-administered questionnaire on anxiety (STAI)

- A questionnaire assessing the behavior of the child (Goodman)

- A questionnaire assessing the child's QoL (VSP-A)

c) The teachers:

- A questionnaire assessing the School Adaptation (GSA questionnaire)

The Cogmed JM group will begin no later than 2 months after the participants' inclusion and the standard group will receive routine management. A similar follow up will be planned for the two groups, based on their intermediate and final visits.
The 8 month (+/- 2 months) intermediate visit

An assessment will be conducted 8 months (+/-2 months) after the participants are included in the study and will consist of:

a) The children

- Assessment of the working memory index (WMI)

- IQ and its main indices (WPSSI IV)

- Auditory attention, statue, and design fluency of the NEPSY-2

- Language and verbal skills (CLéA)

This assessment will be made by a neuropsychologist and will last about 180 minutes.

b) The parents:

- A self-administered questionnaire on anxiety (STAI)

- A questionnaire assessing the behavior of the child (Goodman)

- A questionnaire assessing the child's QoL (VSPA)

c) The teachers: 
- A questionnaire assessing the School Adaptation (GSA questionnaire)

\section{The 18 month (+/- 2 months) final visit}

Children in both the intervention and control groups will receive a final 18 -month evaluation (+/-2 months) after inclusion; they will then be between 7 and $7 \frac{1}{2}$ years old.

The primary evaluation end point: The visuospatial index and the working memory index of the WPPSI-IV will be used for this visit. The WMI will be used to determine the long-term maintenance of the working memory and its impact on intelligence. Auditory attention of NEPSY-2 will be used in this final evaluation. A neuropsychologist will make the assessment that will last about $30 \mathrm{~min}$.

\section{Data management}

Using EpiData software, a specific database will be created. Each participant will be assigned a unique, anonymous number and a data quality control will be performed by a physician to minimize any data inconsistencies.

\section{Statistical methods}

Data will be analyzed using the Statistical Package for the Social Sciences (SPSS) version 20.0 software. Statistical significance is defined as $p<0.05$. The methodology will be based on the Consolidated Standards of Reporting Trials Statement (CONSORT, http:// www.consortstatement.org/consort-statement/) [62].

The full analysis set will be used for the primary analysis. There is no interim analysis planned.

Demographic and baseline characteristics will be summarized. Quantitative data will be shown as mean \pm standard deviation or median with its interquartile range. The qualitative data will be described as percentages with a $95 \%$ confidence interval.

\section{Analysis of primary endpoint}

The mean scores of the VSI will be compared between groups (Student $t$ test or Mann Whitney test). Linear regression will be performed to adjust for potential confounding factors; variables relevant to the models will be selected on their clinical interest and/or a threshold $p$ value $<=0.1$ during bivariate analysis. The final models will express the beta standardized. The unadjusted analysis will be the primary analysis, and the adjusted analysis will be secondary analysis.

Methodology to account for missing data Children not meeting the primary endpoint measurements will be considered as having failed in the study regardless of their randomization group. Additional analyses will be conducted based on:

- Available data and,

- After multiple imputation of missing data.

\section{Analysis of secondary endpoints}

The secondary endpoints will be compared between groups according to the nature of the variable.

The analysis for repeated measurements will be performed to compare changes over time (baseline, eight (+/-2 months) and 18 months (+/- two months) between groups.

Patient selection to include in the analysis All the children registered and randomized in the study will be included in the analysis, respecting the intention to treat. This analysis will be conducted, if necessary, under the maximum bias hypothesis. In a second step, those wrongly included, and any major protocol deviations, will be excluded from the analysis.

\section{Discussion}

This project's primary goal is to demonstrate the necessity of early visuospatial memory assessment within the vulnerable population of VP children, as well as to prove the feasibility and efficacy of computerized CT using online software programs.

Neurodevelopmental problems are common in VP children. Recent publications have reported many disorders in their specific cognitive functions, one of which is WM. Although neuro-development of VP children remains a public health priority because of their increased birth and survival rates, the interventions to improve this are few. There are currently no truly effective interventions to deal with academic achievement in preschoolage VP infants and their neuropsychological problems; thus, currently no specific management care is recommended for these children.

Cogmed is based on interactive software and parental support. The program takes into account the child's environment, a factor of great relevance since it is closely linked to the child's future development. The expected benefits of CT (Cogmed) for those children in this study are enhanced WM, possibly leading to better EF by taking advantage of cerebral plasticity.

A better global neuropsychological development improvement (language and visuospatial processing) can be expected with an improvement in learning and decreased behavioral problems. For parents their guidance in Cogmed helps reduce their anxiety by fully embracing their role as primary agents in their child's development. This is consistent with recommendations for familycentered healthcare and can significantly improve the 
quality of life for both the children and their parents. In the long term, these improvements might also reduce those global costs linked to the consequences of extreme prematurity.

Finally, if proved effective for this vulnerable population, this treatment can be a possible option or an alternative for other preschool populations complaining of early academic difficulties related to WM deficits. Furthermore, the study design based in a randomized, placebo-controlled, is considered as an appropriate design to demonstrate the efficacy of a new experimental intervention in accordance with the Levels of Evidence classification of the Evidence-Based Medicine Working Group [63]. Our findings could be used in the future to update the national and international recommendations concerning very preterm children and training of executive functions.

\section{Abbreviations}

CT: Cognitive Training; EF: Executive Functions; EPIPAGE: Epidemiological study on small gestational ages; GSA: Global School Adaptation score; IQ: Intellectual Quotient; NEPSY-2: NEeuroPSYchology assessment - Second Edition; QoL: Quality of Life; SD: Standard Deviation; STAI: Spielberger StateTrait Anxiety Inventory; VP: Very Preterm; VSI: VisuoSpatial Index; VSPA: Perceived quality of life and health of adolescents and children questionnaire; WM: Working Memory; WMI: Working Memory Index; WPPSIIV: Wechsler Pre-Primary Scale of Intelligence - Fourth Edition

\section{Acknowledgements}

The authors would like to thank all collaborators of EPIREMED-Study Group who participate in the study.

EPIREMED-Study Group:

Meriem ZAHED, Patricia GARCIA, Tristan DESILES, Ludovic ZAHED, Mélodie PACHE, Gwenaëlle MENARD, (AP-HM, France),

Nathalie BEDNAREK WEIRAUCH, Karine VOIRIN, Virginie VERRIERE (University Hospital of Reims, France),

Gilles CAMBONIE, Claire LERAT, Maythé POUJOL (University Hospital of Montpellier, France),

Olivier CLARIS, Sophie RUBIO GURUNG, Eliane BASSON, Melanie RODRIGUEZ, Anne RANNAUD, Johanna BOULANT (University Hospital of Lyon, France), Thierry DEBILLON, Isabelle PIN, Karine GUICHARDET, Caroline TOURNEGROS (University Hospital of Grenoble, France),

Laurence FOIX L'HELIAS, Delphine MITANCHEZ, Jennifer SOMMER, Hélène RUYS MASSON (AP- HP - Armand Trousseau Hospital, France),

Michele GRANIER, Marylène RIOU, Dalia MIGNOT (Sud Francilien Hospital, France),

Bernard GUILLOIS, Valérie DORRIERE DATIN, Mireille DENAVEAUT BOULAY, Delphine ROTS (University Hospital of Caen Normandie, France), Jean-Michel HASCOT, Hélène DEFORGE, Sabine GUIGNON (Regional University Hospital of Nancy, France),

Pierre KUHN, Anne DE SAINT MARTIN, Claire ZORES KOENIG, Hélène MUSMEAUX, Lucille SCHNEIDER, Carole RAMOUSSET, Coralie MANGIN (Regional University Hospital of Strasbourg, France),

Bénédicte LECOMTE, Angélique PANNETIER, Emmanuelle ROCHETTE, Nelly GOUDON-DUBOIS (University Hospital of Clermont-Ferrand, France), Julie OERTEL, Sandrine LA PLANETA (University Hospital of Nice, France), Stéphane MARRET, Marie LEMARCHAND, Nathalie MESTRE (University Hospital of Rouen, France),

Hugues PATURAL, Sophie FLORI (University Hospital of Saint-Etienne, France), Jean-Christophe ROZE, Charlotte COUDRONNIERE, Hamida MARTIN, Alix LAURENT (University Hospital of Nantes, France),

Elie SALIBA, Patrick ZANDER, Eva AOUSTIN (University Hospital of Tour, France),

Catherine ARNAUD, Emeline DUBOIS, Stephanie IANNUZZI, Carine DUFFAUT (University Hospital of Toulouse, France),
Isabelle SOUKSI MEDIONI, Magali REBATTEL, Elodie FALQUE, Nathalie RUMEAU (University Hospital of Nimes, France),

Valérie BENHAMMOU, Laetitia MARCHAND-MARTIN, Samira MEDJAHED (University of Paris, CRESS, Obstetrical Perinatal and Pediatric Epidemiology Research Team, EPOPé, INSERM, INRAE, F-75004 Paris, France)

\section{Authors' contributions}

$C G, P-Y A, J B, M K$ were involved in the conceptualization of the EPIREMED study protocol and wrote this article; KB, MK and ABA were involved in the methodology of the EPIREMED study protocol and wrote the methods section of this article, EPIREMED-Study Group was involved in the validation of the EPIREMED study protocol. All authors were involved in revising the manuscript and have read and approved the final manuscript.

\section{Funding}

The project was funded by a national grant from the French Ministry of Social Affairs and Health (Ministère des Affaires Sociales et de la Santé, grant number: PHRC-15-626). This funding source had no role in the design of this study and will not have any role during its execution, analyses, interpretation of the data, or decision to submit results.

\section{Availability of data and materials}

The datasets that will be generated and/or analyzed during the current study are not publicly available due to the data belongs to the Assistance Publique Hopitaux de Marseille. However, datasets are available from the sponsor (promotion.interne@ap-hm.fr) on reasonable request and after sign a contract pertaining to the provision of data and /or results.

\section{Declarations}

\section{Ethics approval and consent to participate}

The expected sponsor is represented by the Assistance Publique - Hôpitaux de Marseille and must have approvals from the French authorities prior to the study's initiation. The sponsor and the investigators conduct the study in accordance with Good Clinical Practices and the French applicable regulatory requirements (Public Health Act No. 2004-806 of August 9, 2004 on public health policy and its implementing decrees of August 27, 2006 [Code de la Santé Publique, article L.1121-1]), as the applicable privacy requirements and ethical principles outlined in the Declaration of Helsinki. This study was approved by the French Ethics Committee (Comité de Protection des Personnes Sud Méditerranée V) on April 12, 2016 and by the French National Agency for Medicines and Health Products Safety (Agence Nationale de Sécurité du Médicament et des Produits de Santé) on April 18, 2016 (reference number ID-RCB:2016-A00122-49 - Protocol version 2 of 29 Mars 2016). All amendments will be submitted to these two French organizations and communicated to investigators associated by sponsor. The study was registered in ClinicalTrials.gov (NCT02757794).

In accordance with applicable regulations including Good Clinical Practices, and Sponsor's procedures, a Sponsor's monitor will visit the investigator before, during and after the study. Sponsor's monitors will contact the site prior to the start of the study to review with the site staff the protocol, study requirements, and their responsibilities to satisfy regulatory, ethical, and Sponsor's requirements. When reviewing data collection procedures, the discussion will also include identification, agreement and documentation of data items for which the case report form will serve as the source

document. The type and frequency of monitoring are determined using the definition of the level of monitoring based on patient risk and depend on the number of patients included, the rate of inclusions and difficulties encountered during the conduct of the study (procedures approved by the group quality promotion which determines the level of monitoring to be carried out based on risk to the subject-OECD recommendation on the Governance of Clinical Trials, December 2012). In this study, the level of monitoring is rated "minimal" with a risk for the patient level A. The Sponsor's monitor will verify the signed informed consents. If one / multiple consents are not compliant, files will be monitored randomly. All adverse events occurring during clinical trial have to be collected, verified, registered and reported from Day 0 (inclusion day for the participant) until the last day of the study, or as soon as the investigator becomes aware of the AE that he considers linked to the protocol and this up to its resolution.

Any publication must state that Assistance Publique - Hôpitaux de Marseille is the promoter and the study's databases will be co-owned by the promoter 
Assistance Publique - Hôpitaux de Marseille and by the INSERM U1153, EPOPÉ team. The results, as well as all research-related data, must under no circumstances be transmitted to third parties without compensation negotiated beforehand by the Medical Research Branch of the Assistance Publique Hôpitaux de Marseille and/or INSERM U 1153.

All data, results, inventions and discoveries resulting from the study will automatically become the exclusive property of the sponsor which may use this information in the manner deemed suitable with the agreement of the investigator.

The EPIREMED study is part of EPIPAGE 2 and allows us to benefit from the collaboration of those involved in the EPIPAGE 2 cohort's steering committee. The different investigators are involved in the monitoring and care of very premature babies. Their diverse training and responsibilities meet all aspects of our project. These investigators are either pediatricianneonatologists or pediatric neurologists working in type III neonatal units, in neuro-pediatric services for very premature infants's assessment or in a referral center for learning disabilities.

A written informed consent will be obtained from two parents or legal representative to children participating in the study. Protection of the rights will be guaranteed, and anonymity maintained. In accordance with French laws and regulations respectively, all patient records identities will remain confidential. The study's results will be disseminated to patients via a written report in a clear and accessible language.

\section{Consent for publication}

Not applicable.

\section{Competing interests}

The authors declare that they have no competing interests.

\section{Author details}

'Department of Neonatology, North Hospital, APHM University Hospital, Marseille, France. ${ }^{2}$ CEReSS - Health Service Research and Quality of Life Center, Faculty of Medicine, Aix-Marseille University, 27 Bd Jean Moulin, 13385 Marseille, cedex 05, France. ${ }^{3}$ University of Paris, CRESS, Obstetrical Perinatal and Pediatric Epidemiology Research Team, EPOPé, INSERM, INRAE, F-75004 Paris, France. ${ }^{4}$ Obstetrical, Perinatal, and Pediatric Epidemiology Team, Center of Research in Epidemiology and Statistics (U1153), Paris University, INSERM, Paris, France. ${ }^{5}$ Clinical Research Unit, Center for Clinical Investigation P1419, CHU Cochin Broca Hôtel-Dieu, Paris, France.

\section{Received: 16 July 2021 Accepted: 18 August 2021}

\section{Published online: 13 September 2021}

\section{References}

1. Torchin H, Ancel P-Y, Jarreau P-H, Goffinet F. Epidemiology of preterm birth: prevalence, recent trends, short- and long-term outcomes. J Gynecol Obstet Biol Reprod (Paris). 2015;44(8):723-31. https://doi.org/10.1016/j.jgyn.2015.06. 010.

2. Ancel P-Y, Goffinet F, EPIPAGE-2 Writing Group, Kuhn P, Langer B, Matis J, et al. Survival and morbidity of preterm children born at 22 through 34 weeks' gestation in France in 2011: results of the EPIPAGE-2 cohort study. JAMA Pediatr. 2015;169(3):230-8. https://doi.org/10.1001/jamapediatrics.2 014.3351 .

3. Jarjour IT. Neurodevelopmental outcome after extreme prematurity: a review of the literature. Pediatr Neurol. 2015;52(2):143-52. https://doi.org/1 0.1016/j.pediatrneurol.2014.10.027.

4. Doyle LW, Anderson PJ, Battin M, Bowen JR, Brown N, Callanan C, et al. Long term follow up of high risk children: who, why and how? BMC Pediatr. 2014;14(1):279. https://doi.org/10.1186/1471-2431-14-279.

5. Anderson PJ. Neuropsychological outcomes of children born very preterm. Semin Fetal Neonatal Med. 2014;19(2):90-6. https://doi.org/10.1016/j.siny.2 013.11.012.

6. Allotey J, Zamora J, Cheong-See F, Kalidindi M, Arroyo-Manzano D, Asztalos E, et al. Cognitive, motor, behavioural and academic performances of children born preterm: a meta-analysis and systematic review involving 64 061 children. BJOG Int J Obstet Gynaecol. 2018;125(1):16-25. https://doi. org/10.1111/1471-0528.14832.

7. Pierrat V, Marchand-Martin L, Marret S, Arnaud C, Benhammou V, Cambonie $\mathrm{G}$, et al. Neurodevelopmental outcomes at age 5 among children born preterm: EPIPAGE-2 cohort study. BMJ. 2021;373:n741. https://doi.org/10.113 6/bmj.n741.

8. Pierrat V, Marchand-Martin L, Arnaud C, Kaminski M, Resche-Rigon M, Lebeaux $C$, et al. Neurodevelopmental outcome at 2 years for preterm children born at 22 to 34 weeks' gestation in France in 2011: EPIPAGE-2 cohort study. BMJ. 2017;358:j3448. https://doi.org/10.1136/bmj.j3448.

9. Marret S, Chollat C, de Quelen R, Pinto Cardoso G, Abily-Donval L, Chadie A, et al. Course and neurological/behavioral development of preterm children. Arch Pediatr Organe Off Soc Francaise Pediatr. 2015;22:195-202.

10. Ni Y, O'Reilly H, Johnson S, Marlow N, Wolke D. Health-related quality of life from adolescence to adulthood following extremely preterm birth. J Pediatr. 2021. https://doi.org/10.1016/j.jpeds.2021.04.005.

11. Jacob R, Parkinson J. The potential for school-based interventions that target executive function to improve academic achievement: a review. Rev Educ Res. 2015;85(4):512-52. https://doi.org/10.3102/0034654314561338.

12. Anderson PJ, Reidy N. Assessing executive function in preschoolers. Neuropsychol Rev. 2012;22(4):345-60. https://doi.org/10.1007/s11065-0129220-3.

13. Espy KA. Using developmental, cognitive, and neuroscience approaches to understand executive control in young children. Dev Neuropsychol. 2004; 26(1):379-84. https://doi.org/10.1207/s15326942dn2601_1.

14. Diamond A. Executive functions. Annu Rev Psychol. 2013;64(1):135-68. https://doi.org/10.1146/annurev-psych-113011-143750.

15. Mulder H, Pitchford NJ, Hagger MS, Marlow N. Development of executive function and attention in preterm children: a systematic review. Dev Neuropsychol. 2009;34(4):393-421. https://doi.org/10.1080/87565640902964 524.

16. Aarnoudse-Moens CSH, Weisglas-Kuperus N, van Goudoever JB, Oosterlaan J. Meta-analysis of neurobehavioral outcomes in very preterm and/or very low birth weight children. Pediatrics. 2009;124(2):717-28. https://doi.org/10.1 542/peds.2008-2816.

17. Brydges CR, Landes JK, Reid CL, Campbell C, French N, Anderson M Cognitive outcomes in children and adolescents born very preterm: a metaanalysis. Dev Med Child Neurol. 2018;60(5):452-68. https://doi.org/10.1111/ dmen.13685.

18. van Houdt CA, Oosterlaan J, van Wassenaer-Leemhuis AG, van Kaam AH, Aarnoudse-Moens CSH. Executive function deficits in children born preterm or at low birthweight: a meta-analysis. Dev Med Child Neurol. 2019;61(9): 1015-24. https://doi.org/10.1111/dmcn.14213.

19. Twilhaar ES, Wade RM, de Kieviet JF, van Goudoever JB, van Elburg RM, Oosterlaan J. Cognitive outcomes of children born extremely or very preterm since the 1990s and associated risk factors: a Meta-analysis and Meta-regression. JAMA Pediatr. 2018;172(4):361-7. https://doi.org/10.1001/ja mapediatrics.2017.5323.

20. McBryde M, Fitzallen GC, Liley HG, Taylor HG, Bora S. Academic outcomes of school-aged children born preterm: a systematic review and Meta-analysis. JAMA Netw Open. 2020;3(4):e202027. https://doi.org/10.1001/jama networkopen.2020.2027.

21. Réveillon M, Hüppi PS, Barisnikov K. Inhibition difficulties in preterm children: developmental delay or persistent deficit? Child Neuropsychol J Norm Abnorm Dev Child Adolesc. 2018;24(6):734-62. https://doi.org/10.1 080/09297049.2017.1294665.

22. Baddeley A. Working memory. Science. 1992;255(5044):556-9. https://doi. org/10.1126/science.1736359.

23. Alloway TP, Gathercole SE, Pickering SJ. Verbal and visuospatial short-term and working memory in children: are they separable? Child Dev. 2006;77(6): 1698-716. https://doi.org/10.1111/j.1467-8624.2006.00968.x.

24. Anderson PJ, Doyle LW, Victorian Infant Collaborative Study Group. Executive functioning in school-aged children who were born very preterm or with extremely low birth weight in the 1990s. Pediatrics. 2004;114:50-7.

25. Rose SA, Feldman JF, Jankowski JJ, Van Rossem R. The structure of memory in infants and toddlers: an SEM study with full-terms and preterms. Dev Sci. 2011;14(1):83-91. https://doi.org/10.1111/j.1467-7687.2010.00959.x.

26. Espy KA, Stalets MM, McDiarmid MM, Senn TE, Cwik MF, Hamby A. Executive functions in preschool children born preterm: application of cognitive neuroscience paradigms. Child Neuropsychol J Norm Abnorm Dev Child Adolesc. 2002:8(2):83-92. https://doi.org/10.1076/chin.8.2.83.8723.

27. Borradori Tolsa C, Barisnikov K, Lejeune F, Hüppi P. Development of executive functions in preterm children. Arch Pediatr Organe Off Soc Francaise Pediatr. 2014;21:1035-40. 
28. Baron IS, Erickson K, Ahronovich MD, Litman FR, Brandt J. Spatial location memory discriminates children born at extremely low birth weight and latepreterm at age three. Neuropsychology. 2010;24(6):787-94. https://doi.org/1 $0.1037 / \mathrm{a} 0020382$.

29. Omizzolo C, Scratch SE, Stargatt R, Kidokoro H, Thompson DK, Lee KJ, et al. Neonatal brain abnormalities and memory and learning outcomes at 7 years in children born very preterm. Mem Hove Engl. 2014;22(6):605-15. https://doi.org/10.1080/09658211.2013.809765.

30. Bergman Nutley S, Söderqvist S, Bryde S, Thorell LB, Humphreys K, Klingberg T. Gains in fluid intelligence after training non-verbal reasoning in 4-year-old children: a controlled, randomized study. Dev Sci. 2011;14(3):591-601. https://doi.org/10.1111/j.1467-7687.2010.01022.x.

31. Thorell LB, Lindqvist S, Bergman Nutley S, Bohlin G, Klingberg T. Training and transfer effects of executive functions in preschool children. Dev Sci. 2009;12(1):106-13. https://doi.org/10.1111/j.1467-7687.2008.00745.x.

32. Söderqvist S, Nutley SB, Ottersen J, Grill KM, Klingberg T. Computerized training of non-verbal reasoning and working memory in children with intellectual disability. Front Hum Neurosci. 2012;6:271. https://doi.org/10.33 89/fnhum.2012.00271.

33. Shinaver CS, Entwistle PC, Söderqvist S. Cogmed WM training: reviewing the reviews. Appl Neuropsychol Child. 2014;3(3):163-72. https://doi.org/10.1 080/21622965.2013.875314.

34. Kirk HE, Gray K, Riby DM, Cornish KM. Cognitive training as a resolution for early executive function difficulties in children with intellectual disabilities. Res Dev Disabil. 2015;38:145-60. https://doi.org/10.1016/j.ridd.2014.12.026.

35. Melby-Lervåg M, Hulme C. Is working memory training effective? A metaanalytic review. Dev Psychol. 2013;49(2):270-91. https://doi.org/10.1037/a002 8228.

36. Scionti N, Cavallero M, Zogmaister C, Marzocchi GM. Corrigendum: is cognitive training effective for improving executive functions in preschoolers? A Systematic Review and Meta-Analysis. Front Psychol. 2020; 11:410. https://doi.org/10.3389/fpsyg.2020.00410.

37. Spencer-Smith M, Klingberg T. Benefits of a working memory training program for inattention in daily life: a systematic review and meta-analysis. PLoS One. 2015;10(3):e0119522. https://doi.org/10.1371/journal.pone.011 9522.

38. Grunewaldt KH, Løhaugen GCC, Austeng D, Brubakk A-M, Skranes J. Working memory training improves cognitive function in VLBW preschoolers. Pediatrics. 2013;131(3):e747-54. https://doi.org/10.1542/peds.2 012-1965.

39. Grunewaldt KH, Skranes J, Brubakk A-M, Lähaugen GCC. Computerized working memory training has positive long-term effect in very low birthweight preschool children. Dev Med Child Neurol. 2016;58(2):195-201. https://doi.org/10.1111/dmcn.12841

40. Løhaugen GCC, Antonsen I, Håberg A, Gramstad A, Vik T, Brubakk A-M, et al. Computerized working memory training improves function in adolescents born at extremely low birth weight. J Pediatr. 2011;158:555-561.e4.

41. Pascoe L, Roberts G, Doyle LW, Lee K, Thompson DK, Seal ML, et al. Preventing academic difficulties in preterm children: a randomised controlled trial of an adaptive working memory training intervention IMPRINT study. BMC Pediatr. 2013;13(1):144. https://doi.org/10.1186/14 71-2431-13-144.

42. Anderson PJ, Lee KJ, Roberts G, Spencer-Smith MM, Thompson DK, Seal ML, et al. Long-Term Academic Functioning Following Cogmed Working Memory Training for Children Born Extremely Preterm: A Randomized Controlled Trial. J Pediatr. 2018;202:92-97.e4.

43. Shawn Green C, Bavelier D, Kramer AF, Vinogradov S, Ansorge U, Ball KK, et al. Improving methodological standards in behavioral interventions for cognitive enhancement. J Cogn Enhanc. 2019;3(1):2-29. https://doi.org/10.1 007/s41465-018-0115-y.

44. Elvrum A-KG, Andersen GL, Himmelmann K, Beckung E, Öhrvall A-M, Lydersen S, et al. Bimanual fine motor function (BFMF) classification in children with cerebral palsy: aspects of construct and content validity. Phys Occup Ther Pediatr. 2016;36(1):1-16. https://doi.org/10.3109/01942638.2014. 975314.

45. Marois P, Marois M, Pouliot-Laforte A, Vanasse M, Lambert J, Ballaz L. Gross Motor Function Measure Evolution Ratio: Use as a Control for Natural Progression in Cerebral Palsy. Arch Phys Med Rehabil. 2016;97:807-814.e2.

46. Torrioli MG, Frisone MF, Bonvini L, Luciano R, Pasca MG, Lepori R, et al. Perceptual-motor, visual and cognitive ability in very low birthweight preschool children without neonatal ultrasound abnormalities. Brain and
Development. 2000;22(3):163-8. https://doi.org/10.1016/S0387-7604 (00)00098-X.

47. Lecerf T, Kop J-L, Dauvier B. Exploratory network analysis of the French Wechsler intelligence scale for children-fourth edition (WISC-IV). 2017. https://archive-ouverte.unige.ch/unige:98494. Accessed 27 Feb 2018.

48. Korkman M, Kemp SL, Kirk U. Effects of age on neurocognitive measures of children ages 5 to 12: a cross-sectional study on 800 children from the United States. Dev Neuropsychol. 2001;20(1):331-54. https://doi.org/10.1207/ S15326942DN2001_2.

49. van de Weijer-Bergsma E, Wijnroks L, Jongmans MJ. Attention development in infants and preschool children born preterm: a review. Infant Behav Dev. 2008;31(3):333-51. https://doi.org/10.1016/j.infbeh.2007.12.003.

50. Charollais A, Stumpf M-H, De Quelen R, Rondeau S, Pasquet F, Marret S, et al. Delayed language development at two years of age in very preterm infants in the perinatal network of Haute-Normandie. Early Hum Dev. 2014; 90(12):891-2. https://doi.org/10.1016/j.earlhumdev.2014.10.001.

51. Adams AM, Gathercole SE. Limitations in working memory: implications for language development. Int J Lang Commun Disord. 2000;35(1):95-116. https://doi.org/10.1080/136828200247278.

52. Rodrigues A, Befi-Lopes DM. Phonological working memory and its relationship with language development in children. Fono Rev Atualizacao Cient. 2009;21(1):63-8. https://doi.org/10.1590/S0104-56872009000100011.

53. van Noort-van der Spek IL, Franken M-CJP, Weisglas-Kuperus N. Language functions in preterm-born children: a systematic review and meta-analysis. Pediatrics. 2012;129(4):745-54. https://doi.org/10.1542/peds.2011-1728.

54. Reidy N, Morgan A, Thompson DK, Inder TE, Doyle LW, Anderson PJ. Impaired language abilities and white matter abnormalities in children born very preterm and/or very low birth weight. J Pediatr. 2013;162(4):719-24. https://doi.org/10.1016/j.jpeds.2012.10.017.

55. Goodman R, Scott S. Comparing the strengths and difficulties questionnaire and the child behavior checklist: is small beautiful? J Abnorm Child Psychol. 1999;27(1):17-24. https://doi.org/10.1023/A:1022658222914.

56. Simeoni MC, Sapin C, Antoniotti S, Auquier P. Health-related quality of life reported by French adolescents: a predictive approach of health status? J Adolesc Health Off Publ Soc Adolesc Med. 2001;28(4):288-94. https://doi. org/10.1016/S1054-139X(00)00198-1.

57. Ravens-Sieberer U, Auquier P, Erhart M, Gosch A, Rajmil L, Bruil J, et al. The KIDSCREEN-27 quality of life measure for children and adolescents: psychometric results from a cross-cultural survey in 13 European countries. Qual Life Res Int J Qual Life Asp Treat Care Rehabil. 2007;16(8):1347-56. https://doi.org/10.1007/s11136-007-9240-2.

58. Guimard P, Cosnefroy O, Florin A. Évaluation des comportements et des compétences scolaires par les enseignants et prédiction des performances et des parcours à l'école élémentaire et au collège. Orientat Sc Prof. 2007: 179-202. https://doi.org/10.4000/osp.1372.

59. Boussicault G, Nguyen The Tich S, Branger B, Guimard P, Florin A, Rozé J-C, et al. The global school adaptation score: a new neurodevelopmental assessment tool for very preterm children at five years of age. J Pediatr. 2013;163(2):460-4. https://doi.org/10.1016/j.jpeds.2013.01.052.

60. Spielberger CD. Manual for the state-trait anxiety inventory STAI (form Y) ("self-evaluation questionnaire"). 1983. http://ubir.buffalo.edu/xmlui/handle/1 0477/1873. Accessed 8 Apr 2021.

61. Ancel P-Y, Goffinet F, EPIPAGE 2 writing group. EPIPAGE 2: a preterm birth cohort in France in 2011. BMC Pediatr. 2014;14(1):97. https://doi.org/10.11 86/1471-2431-14-97.

62. Schulz KF, Altman DG, Moher D. CONSORT 2010 statement: updated guidelines for reporting parallel group randomised trials. J Pharmacol Pharmacother. 2010;1 (2):100-7. https://doi.org/10.4103/0976-500X.72352.

63. Garg R, Lakhan SE, Dhanasekaran AK. How to review a case report. J Med Case Rep. 2016;10(1):1-5. https://doi.org/10.1186/s13256-016-0853-3.

\section{Publisher's Note}

Springer Nature remains neutral with regard to jurisdictional claims in published maps and institutional affiliations. 